\title{
The Trilateral Evolutionary Game of Agri-Food Quality in Farmer-Supermarket Direct Purchase: A Simulation Approach
}

\author{
Xin Su $\mathbb{D},{ }^{1}$ Haolong Liu $\mathbb{D},{ }^{2}$ and Shunqi Hou $\mathbb{D}^{3}$ \\ ${ }^{1}$ School of MBA, Shandong University of Finance and Economics, Jinan 250014, China \\ ${ }^{2}$ School of Business Administration, Shandong University of Finance and Economics, Jinan 250014, China \\ ${ }^{3}$ College of Management and Economics, Tianjin University, Tianjin 300072, China \\ Correspondence should be addressed to Shunqi Hou; sqhou170228@126.com
}

Received 3 September 2017; Revised 29 November 2017; Accepted 26 December 2017; Published 22 February 2018

Academic Editor: Carlos Gershenson

Copyright (C) 2018 Xin Su et al. This is an open access article distributed under the Creative Commons Attribution License, which permits unrestricted use, distribution, and reproduction in any medium, provided the original work is properly cited.

\begin{abstract}
The prevalence of opportunistic behaviors in agri-food production and circulation results in frequent quality accidents in emerging economies. Numerous researches have discussed effective countermeasures to this problem, but few of them focus on the effectiveness and stability of quality assurance systems. Owing to the bounded rationality and information asymmetry, the dynamic quality game among producers, marketers, and consumers has significant characteristics of complexity. This paper aims at discussing the farmer-supermarket direct purchase's contributions to ensure the agri-food quality and analyzing the effectiveness, stability, and key factors of this new industrial organization. Based on the evolutionary game theory, we establish the trilateralgame payoff matrix, build up the replicator dynamic equations, and discuss possible evolutionary stable states. The simulation results show that the evolutionary system converges to desired stability faster, when the high-quality agri-food's market premium increases and the penalty for violating quality standards increases. Furthermore, when farmers share more high-quality agri-food's market premiums and marketers compensate more for violating the quality standards than before, the evolutionary system also converges to desired stability faster. Therefore, the quality information tracing technology, farmers and marketers' fair distribution of profits and risks, and consumers' capabilities to safeguard their legal rights are the three key factors to maintain the effectiveness and stability of quality assurance systems.
\end{abstract}

\section{Introduction}

The factor and institution resources are less munificent in emerging economies than in the developed ones. So the agrifood supply chain agents in emerging economies tend to take opportunistic behaviors to obtain super-normal profits, resulting in prevalence of quality accidents. Numerous scholars have discussed effective countermeasures to shield innocent consumers from quality opportunistic behaviors within the conceptual frameworks of institutional economics, customer behaviors, game theory, and so forth. It has been revealed that cooperation between producers and marketers would improve and maintain the agri-food quality, but owing to the market risks coming from information asymmetry and consumers' cognitive limitation, the cooperation for improving and maintaining agri-food quality might be vulnerable $[1,2]$. The existing researches draw a key question of how to ensure the cooperation between producers and marketers for quality improvement in such uncertain environments. Fortunately, the industrial organization theory might shed lights on the solution of this question. Farmer-supermarket direct purchase is a new industrial organization mode gradually adopted by Chinese agri-food supply chain, which refers to that supermarkets purchase agri-food of certain quality standards designed by the contracts directly from farmers [3]. This mode would reduce transaction costs, improve operational efficiency, and ensure the quality and safety of agri-food.

The first researching stream of agri-food quality enhancement focuses on the agri-food production. The farmers always face a trade-off between quantity and quality, and reaching higher yields might have strong substitution effects with quality for more productive cultivars [4]. After accepting high-quality production standards, farmers undergo an 
adaptation process in the short-run, where the adjustment to new technologies and practices generates a temporary drop in yields [5]. In contexts of the contract farming, price penalties and bonus payments are effective instruments to ensure consistent high quality, but they both entail additional costs [6]. According to the simulation, the subsidy scheme could promote compliance with high-quality standards and contribute to an upgrade of the agri-food supply chain, but its marginal effectiveness is diminishing [7]. When there exists a collective product reputation without traceability, the producer would extract reputation from the reputation stock when it sells low-quality products at high prices given by the high past levels of quality $[8,9]$. This stream emphasizes the establishment of effective industrial organizations, as well as the designing of effective rewarding and punishment systems, which help guarantee the agri-food quality.

The second researching stream of agri-food quality enhancement focuses on the agri-food consumption. Consumers could not have access to accurate insights into agrifood quality, so quality effects are estimated from the error terms associated with the functions of the unit values, and fresh agri-food would get a higher premium than processed agri-food [10]. Some socioeconomic characteristics have significant impacts on consumer demands for agri-food quality and the elasticity of demands for agri-food quality is inelastic [11]. Consumers of various types have different inferences and evaluations for agri-food quality, which results in significant differences in willingness to purchase high-quality agri-food [12]. Furthermore, the inefficiency of information conduction and feedback in an agri-food value chain would reduce the level of agri-food quality; therefore, the construction and improvement of information platform might facilitate consumers and marketers to adopt a proactive approach to achieve long-term and stable cooperation [13]. This stream emphasizes the vertical integration of agri-food supply chains, which could reinforce long-term trust between grower and buyer and overcome the information asymmetry.

The third researching stream of agri-food quality enhancement focuses on the gaming behaviors of agri-food supply chain agents. Mccluskey [14] discussed consumers and marketers' game behaviors under the condition of information asymmetry when the agri-food is search goods, experience goods, or credence goods. Hoffmann [15] examines the effects of ownership structures on endogenous quality choices and the subsequent equilibrium outcomes within a duopoly framework. Based on a three-stage dynamic game, Fousekis [16] analyzes quality choices in a vertical structure involving a monopolist producer and a monopolist marketer. Based on institutional economics and customer behaviors, numerous scholars discuss the gaming behavior in the production and circulation of agri-food. They discussed the producers' trade-off of quality investment and market premium, as well as the effects of consumers' psychological characteristics on purchasing decision in detail.

Actually, the agri-food quality promotion mechanism in any industrial organization mode is somehow a complex system, which has the distinct properties such as nonlinearity, emergence, spontaneous order, and adaptation. Due to the game agents' bounded rationality and the uncertainty in strategy selection, each game agent chooses better strategies by constant adaptation and learning, leading the evolutionary stable states in the game. In this paper, the payoff matrix of farmers, marketers, and consumers on agri-food quality strategies is established based on the agricultural economics and the evolutionary game theory. And then, the conditions of evolutionary stable states to maintain high-quality standards are discussed. Finally, with the simulation platform, the main conclusions and suggestions are proposed according to the simulation results. The novelty of this paper is reflected in the following two aspects: (1) evolutionary game methods are applied to analyze the agri-food quality promotion mechanism of a new industrial organization mode, namely, farmersupermarket direct purchase; (2) the nonlinearity, emergence, spontaneous order, adaptation, and other complexity characteristics of agri-food quality promotion mechanism are revealed with the simulation platform, which might address the theoretical gap resulting from static analysis.

\section{Model}

According to the agricultural economics and game theory, it is assumed that there are three kinds of participants in the agri-food quality evolutionary game. The first group (namely, group A) is the farmers which are engaged in the production of agri-food in rural areas. The second group (namely, group B) is the marketers which are engaged in sales of agrifood in downtown. The third group (namely, group C) is the consumers which infer the agri-food quality and decide whether to trust the marketers and buy the agri-food. Besides, there is no intermediary organization or agent among these groups, meaning that the marketers purchase agri-food of certain quality standards from the rural farmers according to the contracts signed previously, and then they sell agri-food to customers directly and randomly.

There are 2 optional strategies for group $A$ : the first one is to provide high-quality agri-food that abided by the quality criteria $\left(S_{A 1}\right)$; the second one is to provide low-quality agrifood that violates the quality criteria $\left(S_{A 2}\right)$. Similarly, there are 2 optional strategies for group $B$ : the first one is to purchase and sell high-quality agri-food $\left(S_{B 1}\right)$; the other is to purchase and sell low-quality agri-food $\left(S_{B 2}\right)$. The group $C$ also has 2 optional strategies: the former is to buy $\left(S_{C 1}\right)$ and the latter is not to buy $\left(S_{C 2}\right)$. According to the game tree, eight strategy combinations are concluded as in Figure 1.

$C_{H}$ and $C_{L}$, respectively, represent group $A$ 's production costs to provide high-quality and low-quality agri-food [17]. $W_{H}$ and $W_{L}$, respectively, represent group $B$ 's procurement costs to implement high-quality and low-quality standards. $P_{H}$ and $P_{L}$, respectively, represent group $B$ 's equilibrium prices to sell high-quality and low-quality agri-food [18]. $U_{H}$ and $U_{L}$, respectively, represent group $C$ 's utilities to consume high-quality and low-quality agri-food. According to the actual situation in emerging economies, it is assumed that $C_{H}>C_{L}, W_{H}>W_{L}, P_{H}>P_{L}$, and $U_{H}>U_{L}$. It is also assumed that $W_{H}-C_{H}<W_{L}-C_{L}, P_{H}-W_{H}<P_{L}-W_{L}$ but $U_{H}-P_{H}>U_{L}-P_{L}$. Therefore, the farmers' marginal revenue to produce high-quality agri-food is less than the marginal 


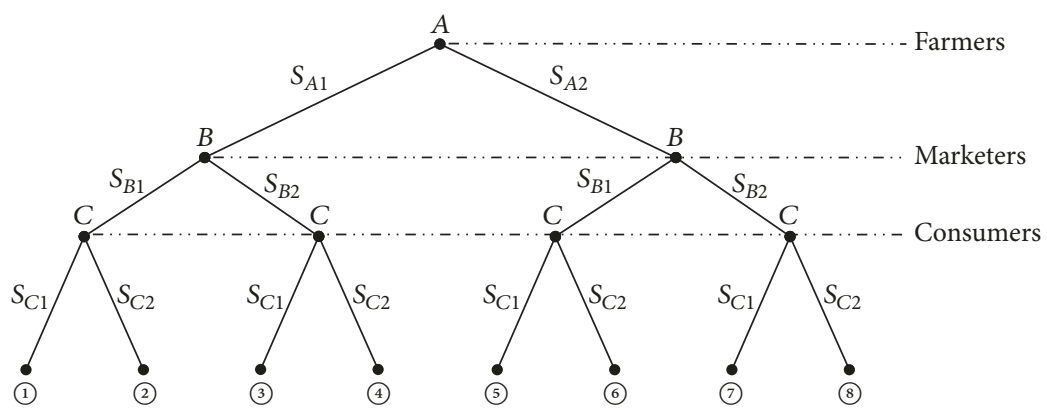

FIgURE 1: The game tree of agri-food quality.

revenue they produce low-quality ones, leading to their frequently violating the quality criteria. Moreover, in agrifood markets of emerging economies, the marketer would get lower marginal revenue for selling agri-food with high quality. In addition, eating the low-quality agri-food would have considerable negative impact on consumers' utility, satisfaction, and even health.

In strategy combination (1), when groups $A, B$, and $C$ all choose strategy 1 , the marketers could gain a market premium $R$ that comes from quality certification, customer satisfaction, customer loyalty, and so forth. In the ordinary way, marketers do not monopolize market premium $R$ and they would deliver a part of the value to farmers, in order to maintain a long-term cooperative relationship and to keep farmers from breaking quality contracts due to the low marginal revenue. The market premium delivered to farmers is $R_{A}$, which equals $\delta * R$ and the market premium retained by marketers is $R_{B}$ which equals $R-R_{A}$. In strategy combination (2), when groups $A$ and $B$ choose strategy 1 but group $C$ chooses strategy 2 , consumers' utility is 0 and marketers could not gain the market equilibrium price $P_{H}$ nor the market premium $R$.

In strategy combination (3), when groups $A$ and $C$ choose strategy 1 but group $B$ chooses strategy 2 , although farmers pay higher production cost $C_{H}$, they only gain lower procurement price $W_{L}$ because the marketers refuse to implement the high-quality standard. When consumers buy low-quality agri-food at a lower market equilibrium price $P_{L}$ and gain undesirable utility after eating low-quality agri-food, they might report to supervision departments and charge the marketers with low-quality standards. Actually, the probability of charging illegal marketers successfully depends on consumers' awareness, capabilities, and conditions to safeguard their legal rights, so it is assumed that the mathematical expectation of compensation is $K$. In strategy combination (4), marketers could not gain the lower market equilibrium price $P_{L}$ but they could be exempt from administrative penalty.

In strategy combination (5), when groups $B$ and $C$ choose strategy 1 but group $A$ chooses strategy 2 , farmers pay lower production cost $C_{L}$ but gain higher procurement price $W_{H}$, because the marketers implement high-quality standards for maintaining their market presence and competitive advantages. However, the marketers are bound to perceive farmers' opportunistic behaviors by quality monitoring and farmers would pay a fine of $D$ for violating the quality criteria. The marketers have to seek new suppliers or take additional quality improvement measures for maintaining the high quality of agri-food, which brings extra transactional and operational costs $E$ [19]. Usually the amount of the fine is determined by the marketers, which just compensates the extra cost $(D=E)$. In addition, according to $W_{H}-C_{L}-D<$ $W_{H}-C_{H}$, the marketers monopolize the quality premium $R$ [20]. In strategy combination (6), the marketers could not gain the equilibrium price $P_{H}$ nor the market premium $R$, although they pay extra transactional and operational costs E.

In strategy combination ( 7 , when groups $A$ and $B$ choose strategy 2 but group $C$ chooses strategy 1 , the consumers would demand compensation $K$ after having the agri-food with low quality, and both farmers and marketers assume compensation liability because they both break high-quality standards of production and sales. The indemnity paid by farmers is $K_{A}$ which equals $\varepsilon * K$ and the indemnity paid by marketers is $K_{B}$ which equals $K-K_{A}$. In strategy combination (8), the marketers could not gain the equilibrium price $P_{L}$ but they could be exempt from administrative penalty.

It is assumed that the percentages of the farmers, marketers and consumers choosing strategy 1 are $x, y$ and $z$. Similarly the percentages of the farmers, marketers, and consumers choosing strategy 2 are $1-x, 1-y$, and $1-z$. Therefore, the complete payoff matrix of farmers, marketers, and consumers on agri-food quality strategies is established as in Table 1.

\section{Discussion}

3.1. Replicator Dynamics. The replicator dynamics is an explicit model of a selection process, specifying how population shares associated with different pure strategies in a game evolve over time [21]. According to evolutionary game theory, if the fitness of a certain adaptive strategy or expected revenue is higher than the average fitness, the proportion of the participants choosing this strategy would gradually increase to a specific population. Because the information about the gaming results would be disseminated and shared in whole population, the group with lower revenue would gradually optimize strategic choosing with bounded rationality. The growth rate is determined by the replicator dynamic differential equations. The higher value of replicator dynamics is, the 
TABLE 1: Payoff matrix of agri-food quality game.

\begin{tabular}{lccc}
\hline Strategy combination & Group A's payoff & Group B's payoff & Group C's payoff \\
\hline (1) $1,1,1$ & $-C_{H}+W_{H}+R_{A}$ & $-W_{H}+P_{H}+R_{B}$ & $-P_{H}+U_{H}$ \\
(2) $1,1,2$ & $-C_{H}+W_{H}$ & $-W_{H}$ & 0 \\
(3) $1,2,1$ & $-C_{H}+W_{L}$ & $-W_{L}+P_{L}-K$ & $-P_{L}+U_{L}+K$ \\
(4) $1,2,2$ & $-C_{H}+W_{L}$ & $-W_{L}$ & 0 \\
(5) $2,1,1$ & $-C_{L}+W_{H}-D$ & $-W_{H}+D-E+P_{H}+R$ & $-P_{H}+U_{H}$ \\
(6) $2,1,2$ & $-C_{L}+W_{H}-D$ & $-W_{H}+D-E$ & 0 \\
(7) $2,2,1$ & $-C_{L}+W_{L}-K_{A}$ & $-W_{L}+P_{L}-K_{B}$ & $-P_{L}+U_{L}+K$ \\
(8) $2,2,2$ & $-C_{L}+W_{L}$ & $-W_{L}$ & 0 \\
\hline
\end{tabular}

Note. The proportions of groups $A, B$, and $C$ choosing strategy 1 are $x, y$, and $z$.

faster the proportion will increase. According to the payoff matrix, the fitness (expected revenue) of group $A$ choosing strategy 1, group $A$ choosing strategy 2 , and group $A$ choosing mixed strategies could be calculated.

$$
\begin{aligned}
U_{A 1} & =\left[-C_{H}+W_{L}\right]+y\left[W_{H}-W_{L}\right]+y z R_{A} \\
U_{A 2} & =\left[-C_{L}+W_{L}\right]+y\left[W_{H}-W_{L}-D\right]+(1-y) z K_{A} \\
U_{A E} & =x U_{A 1}+(1-x) U_{A 2} .
\end{aligned}
$$

$U_{A 1}$ represents the expected revenue of group $A$ choosing strategy $1 ; U_{A 2}$ represents the expected revenue of group $A$ choosing strategy $2 ; U_{A E}$ represents the average fitness. Therefore, we could establish the replicator dynamic equation of group $A$ choosing strategy $1, F_{A}$. For simplicity and to facilitate the subsequent analysis, we introduce $G_{A}$ into the replicator dynamic equation, which is a function of $y$ and $z$.

$$
\begin{aligned}
F_{A} & =\frac{d x}{d t}=U_{A 1}-U_{A E}=x(1-x)\left(U_{A 1}-U_{A 2}\right) \\
& =x(1-x) G_{A} \\
G_{A} & =\left[-C_{H}+C_{L}\right]+y D-z K_{A}+y z R_{A}+y z K_{A} .
\end{aligned}
$$

Similarly, the fitness of group $B$ choosing strategy 1 , group $B$ choosing strategy 2 , and group $B$ choosing mixed strategies could be calculated according to the payoff matrix. $U_{B 1}$ represents the expected revenue of group $B$ choosing strategy $1 ; U_{B 2}$ represents the expected revenue of group $B$ choosing strategy $2 ; U_{B E}$ represents the average fitness.

$$
\begin{aligned}
U_{B 1}= & {\left[-W_{H}+D-E\right]+x[-D+E]+z\left[P_{H}+R\right] } \\
& +x z\left[R_{B}-R\right] \\
U_{B 2}= & {\left[-W_{L}\right]+z\left[P_{L}-K_{B}\right]+x z\left[K_{B}-K\right] } \\
U_{B E}= & y U_{B 1}+(1-y) U_{B 2} .
\end{aligned}
$$

Therefore, we could establish the replicator dynamic equation of group $B$ choosing strategy $1, F_{B}$. For simplicity and to facilitate the subsequent analysis, we introduce $G_{B}$ into the replicator dynamic equation, which is a function of $x$ and $z$.

$$
\begin{aligned}
F_{B}= & \frac{d y}{d t}=U_{B 1}-U_{B E}=y(1-y)\left(U_{B 1}-U_{B 2}\right) \\
= & y(1-y) G_{B} \\
G_{B}= & {\left[-W_{H}+W_{L}\right]+z\left[P_{H}-P_{L}+R+K_{B}\right] } \\
& +x z\left[-R+R_{B}+K-K_{B}\right] .
\end{aligned}
$$

Furthermore, the fitness of group $C$ choosing strategy 1 , group $C$ choosing strategy 2 , and group $C$ choosing mixed strategies could be calculated according to the payoff matrix. $U_{C 1}$ represents the expected revenue of group $C$ choosing strategy $1 ; U_{C 2}$ represents the expected revenue of group $C$ choosing strategy $2 ; U_{C E}$ represents the average fitness.

$$
\begin{aligned}
U_{C 1}= & {\left[-P_{L}+U_{L}+K\right] } \\
& +y\left[-P_{H}+P_{L}+U_{H}-U_{L}-K\right] \\
U_{C 2}= & 0 \\
U_{C E}= & z U_{C 1}+(1-z) U_{C 2} .
\end{aligned}
$$

Therefore, we could establish the replicator dynamic equation of group $C$ choosing strategy $1, F_{C}$. For simplicity and to facilitate the subsequent analysis, we introduce $G_{C}$ into the replicator dynamic equation, which is a function of $y$.

$$
\begin{aligned}
F_{C}= & \frac{d z}{d t}=U_{C 1}-U_{C E}=z(1-z)\left(U_{C 1}-U_{C 2}\right) \\
= & z(1-z) G_{C} \\
G_{C}= & {\left[-P_{L}+U_{L}+K\right] } \\
& +y\left[-P_{H}+P_{L}+U_{H}-U_{L}-K\right] .
\end{aligned}
$$

3.2. Saddle Point. When $G_{A}=G_{B}=G_{C}=0, x=x^{*}, y=$ $y^{*}$ and $z=z^{*}$, and the spatial coordinates are $\left(x^{*}, y^{*}, z^{*}\right)$, 
there is no difference in payoff for each game agent and this point is the saddle point of the evolutionary game.

$$
\begin{aligned}
G_{A} & =0, \\
z^{*} & =\frac{C_{H}-C_{L}-y \cdot D}{y \cdot\left(R_{A}+K_{A}\right)-K_{A}} \in[0,1] \\
G_{B} & =0, \\
x^{*} & =\frac{W_{H}-W_{L}-z \cdot\left(P_{H}-P_{L}+R+K_{B}\right)}{z \cdot\left(K_{A}-R_{A}\right)} \in[0,1] \\
G_{C} & =0, \\
y^{*} & =\frac{-\left(U_{L}-P_{L}+K\right)}{\left(U_{H}-P_{H}\right)-\left(U_{L}-P_{L}+K\right)} \in[0,1] .
\end{aligned}
$$

When $G_{C}=0$, as the utility $U_{L}$ of consumers to have lowquality agri-food is considerably negative, the compensation $K$ claimed by consumers could not completely make up for the utility loss.

$$
U_{H}-P_{H}>0>U_{L}-P_{L}+K
$$

When $G_{A}=0$, we should discuss two possible situations where the numerator is larger than 0 and where the numerator is smaller than 0 , which would be of great advantage for the judgment of evolutionary stable strategy and simulation.

when $C_{H}-C_{L}-y \cdot D>0$,

$$
\max \left\{\frac{K_{A}}{R_{A}+K_{A}}, \frac{K_{A}+C_{H}-C_{L}}{R_{A}+K_{A}+D}\right\}<y<\frac{C_{H}-C_{L}}{D}
$$

when $C_{H}-C_{L}-y \cdot D<0$,

$$
\frac{C_{H}-C_{L}}{D}<y<\min \left\{\frac{K_{A}}{R_{A}+K_{A}}, \frac{K_{A}+C_{H}-C_{L}}{R_{A}+K_{A}+D}\right\} .
$$

When $G_{B}=0$, we should also discuss two possible situations where $K_{A}$ is larger than $R_{A}$ and $K_{A}$ is smaller than $R_{A}$.

$$
\begin{aligned}
\text { when } K_{A}>R_{A} \text {, } \\
\qquad \frac{W_{H}-W_{L}}{P_{H}-P_{L}+R_{B}+K}<z<\frac{W_{H}-W_{L}}{P_{H}-P_{L}+R+K_{B}}
\end{aligned}
$$

when $K_{A}<R_{A}$,

$$
\frac{W_{H}-W_{L}}{P_{H}-P_{L}+R+K_{B}}<z<\frac{W_{H}-W_{L}}{P_{H}-P_{L}+R_{B}+K} .
$$

3.3. Evolutionary Stable Strategy. According to stability theory of differential equation, it is feasible to judge whether a local equilibrium point represents the evolutionary stability with the eigenvalue of the Jacobian matrix. If a local equilibrium point meets the condition that the determinant of Jacobian matrix (Det $J$ ) is positive and the trace of Jacobian matrix $(\operatorname{Tr} J)$ is negative, it would be the evolutionary stable strategy [21] (Friedman, 1998). The judging criteria of evolutionary stable strategies could be formulated as follows.

$$
J=\left[\begin{array}{ll}
\frac{\partial F_{1}}{\partial x_{1}} & \frac{\partial F_{1}}{\partial x_{2}} \\
\frac{\partial F_{2}}{\partial x_{1}} & \frac{\partial F_{2}}{\partial x_{2}}
\end{array}\right]=\left[\begin{array}{ll}
a_{11} & a_{12} \\
a_{21} & a_{22}
\end{array}\right]
$$

$$
\begin{aligned}
\text { Det } J & =a_{11} \cdot a_{22}-a_{12} \cdot a_{21}>0 \\
\operatorname{Tr} J & =a_{11}+a_{22}<0 .
\end{aligned}
$$

But the judging criteria of evolutionary stable strategies do not deal with interactions that take place between more than two individuals at a time [21], which brings difficulties to judge evolutionary stable strategies (ESS) in a trilateral game. To work out this problem, we analyze the conditions of evolutionary stability in pairs and discuss possible evolutionary stable strategies. Because there are two logical stages in the agri-food supply chain, including the wholesaling stage and the retailing stage, we first discuss the possible evolutionary stable strategies in the wholesale procedure and then we discuss the possible evolutionary stable strategies in the interactions between supermarkets and consumers [22]. When the former evolutionary stable strategies are in correspondence with the latter ones, the evolutionary stable strategies of the trilateral evolutionary game in whole process are found. The complete Jacobian matrix and its eigenvalues are formulated as follows.

$$
\begin{aligned}
J= & {\left[\begin{array}{lll}
\frac{\partial F_{A}}{\partial x} & \frac{\partial F_{A}}{\partial y} & \frac{\partial F_{A}}{\partial z} \\
\frac{\partial F_{B}}{\partial x} & \frac{\partial F_{B}}{\partial y} & \frac{\partial F_{B}}{\partial z} \\
\frac{\partial F_{C}}{\partial x} & \frac{\partial F_{C}}{\partial y} & \frac{\partial F_{C}}{\partial z}
\end{array}\right]=\left[\begin{array}{lll}
a_{11} & a_{12} & a_{13} \\
a_{21} & a_{22} & a_{23} \\
a_{31} & a_{32} & a_{33}
\end{array}\right] } \\
a_{11} & =\frac{\partial F_{A}}{\partial x}=(1-2 x)\left\{-C_{H}+C_{L}+y D+y z R_{A}\right. \\
& \left.-(1-y) z K_{A}\right\} \\
a_{12} & =\frac{\partial F_{A}}{\partial y}=x(1-x)\left\{D+z R_{A}+z K_{A}\right\} \\
a_{13} & =\frac{\partial F_{A}}{\partial z}=x(1-x)\left\{y R_{A}-(1-y) K_{A}\right\} \\
a_{21} & =\frac{\partial F_{B}}{\partial x}=y(1-y)\left\{z\left[\left(R_{B}-R\right)-\left(K_{B}-K\right)\right]\right\} \\
a_{22} & =\frac{\partial F_{B}}{\partial y}=(1-2 y)\left\{-W_{H}+W_{L}\right. \\
& \left.+z\left[P_{H}-P_{L}+R+K_{B}\right]+x z\left[R_{B}-R-K_{B}+K\right]\right\} \\
a_{23}= & \frac{\partial F_{B}}{\partial z}=y(1-y)\left\{\left[P_{H}-P_{L}+R+K_{B}\right]\right. \\
+ & \left.x\left[R_{B}-R-K_{B}+K\right]\right\}
\end{aligned}
$$


TABLE 2: Main eigenvalues of Jacobian matrix.

\begin{tabular}{lccccc}
\hline$x, y, z$ & $a_{11}$ & \pm & $a_{22}$ & \pm & $a_{33}$ \\
\hline $1,1,1$ & $-\left\{C_{L}-C_{H}+D+R_{A}\right\}$ & - & $-\left\{W_{L}-W_{H}+P_{H}-P_{L}+R_{B}+K\right\}$ & - & $-\left\{U_{H}-P_{H}\right\}$ \\
$1,1,0$ & $-\left\{C_{L}-C_{H}+D\right\}$ & - & $-\left\{W_{L}-W_{H}\right\}$ & + & - \\
$1,0,1$ & $-\left\{C_{L}-C_{H}-K_{A}\right\}$ & + & $\left\{W_{L}-W_{H}+P_{H}-P_{L}+R_{B}+K\right\}$ & + & $-\left\{U_{L}-P_{L}+K\right\}$ \\
$1,0,0$ & $-\left\{C_{L}-C_{H}\right\}$ & + & $\left\{W_{L}-W_{H}\right\}$ & - & + \\
$0,1,1$ & $\left\{C_{L}-C_{H}+D+R_{A}\right\}$ & + & $-\left\{W_{L}-W_{H}+P_{H}-P_{L}+R_{B}+K\right\}$ & - & $\left.-P_{L}+K\right\}$ \\
$0,0,1$ & $\left\{C_{L}-C_{H}+D\right\}$ & + & $-\left\{W_{L}-W_{H}\right\}$ & + & - \\
$0,1,0$ & $\left\{C_{L}-C_{H}-K_{A}\right\}$ & - & $\left\{W_{L}-W_{H}+P_{H}-P_{L}+R_{B}+K\right\}$ & + & $-\left\{U_{L}\right\}$ \\
$0,0,0$ & $\left\{C_{L}-C_{H}\right\}$ & - & $\left\{W_{L}-W_{H}\right\}$ & $-K\}$ & + \\
\hline
\end{tabular}

Note. $a_{12}=a_{13}=a_{21}=a_{23}=a_{31}=a_{32}=0$.

TABLE 3: The ESS in the first stage.

\begin{tabular}{|c|c|c|c|c|c|c|c|c|}
\hline & $x, y$ & $a_{11}$ & \pm & $a_{22}$ & \pm & Det & $\operatorname{Tr}$ & Stability \\
\hline \multirow{4}{*}{$z=1$} & 1,1 & $-\left\{C_{L}-C_{H}+D+R_{A}\right\}$ & - & $-\left\{W_{L}-W_{H}+P_{H}-P_{L}+R_{B}+K\right\}$ & - & + & - & Stable \\
\hline & 1,0 & $-\left\{C_{L}-C_{H}-K_{A}\right\}$ & + & $\left\{W_{L}-W_{H}+P_{H}-P_{L}+R_{B}+K\right\}$ & + & + & + & Unstable \\
\hline & 0,1 & $\left\{C_{L}-C_{H}+D+R_{A}\right\}$ & + & $-\left\{W_{L}-W_{H}+P_{H}-P_{L}+R_{B}+K\right\}$ & - & - & & Unstable \\
\hline & 0,0 & $\left\{C_{L}-C_{H}+D\right\}$ & + & $-\left\{W_{L}-W_{H}\right\}$ & + & + & + & Unstable \\
\hline \multirow{4}{*}{$z=0$} & 1,1 & $-\left\{C_{L}-C_{H}+D\right\}$ & - & $-\left\{W_{L}-W_{H}\right\}$ & + & - & & Unstable \\
\hline & 1,0 & $-\left\{C_{L}-C_{H}\right\}$ & + & $\left\{W_{L}-W_{H}\right\}$ & - & - & & Unstable \\
\hline & 0,1 & $\left\{C_{L}-C_{H}-K_{A}\right\}$ & - & $\left\{W_{L}-W_{H}+P_{H}-P_{L}+R_{B}+K\right\}$ & + & - & & Unstable \\
\hline & 0,0 & $\left\{C_{L}-C_{H}\right\}$ & - & $\left\{W_{L}-W_{H}\right\}$ & - & + & - & Stable \\
\hline
\end{tabular}

TABLE 4: The ESS in the second stage.

\begin{tabular}{|c|c|c|c|c|c|c|c|c|}
\hline & $y, z$ & $a_{22}$ & \pm & $a_{33}$ & \pm & det & $\operatorname{tr}$ & Stability \\
\hline \multirow{4}{*}{$x=1$} & 1,1 & $-\left\{W_{L}-W_{H}+P_{H}-P_{L}+R_{B}+K\right\}$ & - & $-\left\{U_{H}-P_{H}\right\}$ & - & + & - & Stable \\
\hline & 1,0 & $-\left\{W_{L}-W_{H}\right\}$ & + & $\left\{U_{H}-P_{H}\right\}$ & + & + & + & Unstable \\
\hline & 0,1 & $\left\{W_{L}-W_{H}+P_{H}-P_{L}+R_{B}+K\right\}$ & + & $-\left\{U_{L}-P_{L}+K\right\}$ & + & + & + & Unstable \\
\hline & 0,0 & $\left\{W_{L}-W_{H}\right\}$ & - & $\left\{U_{L}-P_{L}+K\right\}$ & - & + & - & Stable \\
\hline \multirow{4}{*}{$x=0$} & 1,1 & $-\left\{W_{L}-W_{H}+P_{H}-P_{L}+R_{B}+K\right\}$ & - & $-\left\{U_{H}-P_{H}\right\}$ & - & + & - & Stable \\
\hline & 0,1 & $-\left\{W_{L}-W_{H}\right\}$ & + & $\left\{U_{H}-P_{H}\right\}$ & + & + & + & Unstable \\
\hline & 1,0 & $\left\{W_{L}-W_{H}+P_{H}-P_{L}+R_{B}+K\right\}$ & + & $-\left\{U_{L}-P_{L}+K\right\}$ & + & + & - & Unstable \\
\hline & 0,0 & $\left\{W_{L}-W_{H}\right\}$ & - & $\left\{U_{L}-P_{L}+K\right\}$ & - & + & + & Stable \\
\hline
\end{tabular}

$$
\begin{aligned}
& a_{31}=\frac{\partial F_{C}}{\partial x}=0 \\
& a_{32}=\frac{\partial F_{C}}{\partial y}=z(1-z)\left\{P_{L}-P_{H}+U_{H}-U_{L}-K\right\} \\
& a_{33}=\frac{\partial F_{C}}{\partial z}=(1-2 z)\left\{-P_{L}+U_{L}+K\right\} .
\end{aligned}
$$

For generality and simplicity, the main eigenvalues of the complete Jacobian matrix are formulated in Table 2 concerning the strategy combinations. It deserves to be mentioned that $a_{12}, a_{13}, a_{21}, a_{23}, a_{31}$, and $a_{32}$ equal zero.

According to Table 3, under the condition that $z$ equals one, when $F_{A}=0$ and $F_{B}=0$, there are five possible local equilibrium points of the evolutionary game in the plane $\{(x, y) \mid 0 \leq x, y \leq 1\}$, including $(0,0),(0,1),(1,0),(1,1)$, and $\left(x^{*}, y^{*}\right)$. The point $\left(x^{*}, y^{*}\right)$ is the saddle point which is subject to the condition that $G_{A}=G_{B}=0$. According to the judging conditions of evolutionary stability strategy, it is inferred that only the point $(x, y)=(1,1)$ represents the evolutionary stable state of the evolutionary game and the others are unstable points. Similarly, under the condition that $z$ equals zero, the point $(x, y)=(0,0)$ represents the evolutionary stable state of the evolutionary game.

According to Table 4, under the condition that $x$ equals one and $F_{B}=0$ and $F_{C}=0$, there are five possible local equilibrium points of the evolutionary game in the plane $\{(y, z) \mid 0 \leq y, z \leq 1\}$, including $(0,0),(0,1),(1,0),(1,1)$, and $\left(y^{*}, z^{*}\right)$. The point $\left(y^{*}, z^{*}\right)$ is the saddle point which is subject to the condition that $G_{B}=G_{C}=0$. According to the judging conditions of evolutionary stable strategy, it is inferred that points $(1,1)$ and $(0,0)$ represent the evolutionary stable state of the evolutionary game. The point $\left(y^{*}, z^{*}\right)$ is the saddle point and the others are unstable points. Similarly, under the condition that $x$ equals 0 , points $(1,1)$ and $(0,0)$ still 
TABLE 5: Initial values of parameters.

\begin{tabular}{lccc}
\hline Variable & Parameter & Meaning & Initial value \\
\hline$C_{H}$ & 1 & Farmers' production cost to produce high-quality agri-food \\
$C_{L}$ & $\alpha$ & Farmers' production cost to produce low-quality agri-food & 1.000 \\
$W_{H}$ & $(1+w)$ & Wholesale price of high-quality agri-food & 0.500 \\
$W_{L}$ & $(1+w) \beta$ & Wholesale price of low-quality agri-food & 1.500 \\
$P_{H}$ & $(1+p)$ & Retail price of high-quality agri-food & 1.200 \\
$P_{L}$ & $(1+p) \lambda$ & Retail price of low-quality agri-food & 2.500 \\
$U_{H}$ & $u$ & Consumers' utility having high-quality agri-food & 2.250 \\
$U_{L}$ & $v$ & Consumers' utility having low-quality agri-food & 4.500 \\
$D$ & $d$ & Farmers' indemnity for marketers with high-quality criteria \\
$R$ & $(1+p) r$ & Market premium for selling high-quality agri-food \\
$K$ & $(1+p) k$ & Compensation for selling low-quality agri-food \\
$R_{A}$ & $(1+p) r \delta$ & Market premium shared by farmers \\
$R_{B}$ & $(1+p) r(1-\delta)$ & Market premium shared by marketers \\
$K_{A}$ & $(1+p) k \varepsilon$ & Compensation assumed by farmers \\
$K_{B}$ & $(1+p) k(1-\varepsilon)$ & Compensation assumed by marketers & 0.900 \\
\hline
\end{tabular}

represent the evolutionary stable states of the evolutionary game.

As the discussion in Tables 3 and 4, an evolutionary stable strategy in trilateral evolutionary game needs to be subject to the local stable conditions in both stages. Therefore, we could draw the conclusion that only strategy combination $(1,1,1)$ and strategy combination $(0,0,0)$ present the final evolutionary stable states.

\section{Simulation}

For simplicity and generality, we assume group $A$ 's production cost to provide high-quality agri-food $C_{H}$ is 1 and the production cost to provide low-quality agri-food $C_{L}$ is $\alpha(0<$ $\alpha<1)$. Moreover, it is assumed that the wholesale price of high-quality agri-food is $W_{H}$ which equals $1+w(w>0)$ and the wholesale price of low-quality agri-food is $W_{L}$ which equals $(1+w) \beta(0<\beta<1)$. Furthermore, the retail price $P_{H}$ equals $1+p(p>0)$ and $P_{L}$ equals $(1+p) \lambda(0<\lambda<1)$.

The group $B$ 's additional cost $D$ for maintaining a highquality strategy is a function of farmers' production cost to produce high-quality agri-food and it equals $d$. The highquality market premium $R$ is a function of the retail price and it equals $(1+p) r$. Similarly, low-quality market indemnity $K$ is also a function of the retail price and it equals $(1+p) k$. When the customers consume the high-quality agri-food, they gain the utility of $u$ and they would gain the utility of $v$ after consuming the low-quality agri-food.

When both farmers and marketers abide by the highquality standards of production and sales, they would share the market premium and the proportion delivered to farmers is $\delta$. When both farmers and marketers break the high-quality standards of production and sales, they assume compensation liability for consumers together and the proportion assumed by farmers is $\varepsilon$.

To meet the domain of definition of $x, y$, and $z$, the initial values of parameters mentioned above are listed in Table 5,

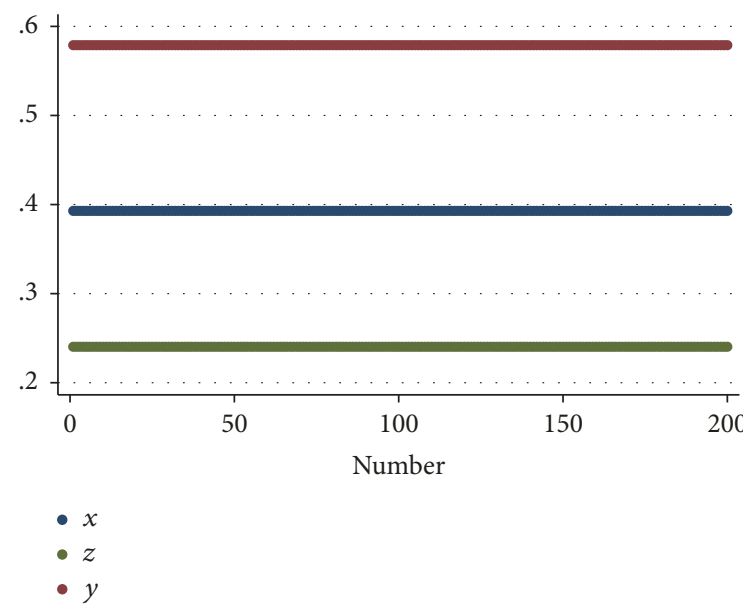

FIGURE 2: Simulation results when initial coordinates coincide with the saddle point.

which is also basically consistent with the market situation in emerging economies [23]. At this time, the saddle point of the evolutionary game is $(0.393,0.579$, and 0.240$)$. And the iteration times are set as 200 in the Netlogo simulation platform.

When the initial value of $(x, y$, and $z)$ is set as $(0.393$, 0.579 , and 0.240$)$, which just coincides with the saddle point in the initial condition, the proportions of participants who choose strategy 1 in each group would undergo no changes after 200 iterations, as shown in Figure 2. When the initial position is above the saddle point, the proportions of participants who choose strategy 1 in each group converge to 1 gradually; when the initial position is under the saddle point, they converge to 0 gradually.

When the initial value of $(x, y, z)$ is set as $(0.40,0.59$, 0.25 ), which is slightly higher than the saddle point, the system would converge to the desirable evolutionary stable state $(1,1,1)$ as shown in the Figure $3(\mathrm{a})$. But when the initial 




(a)

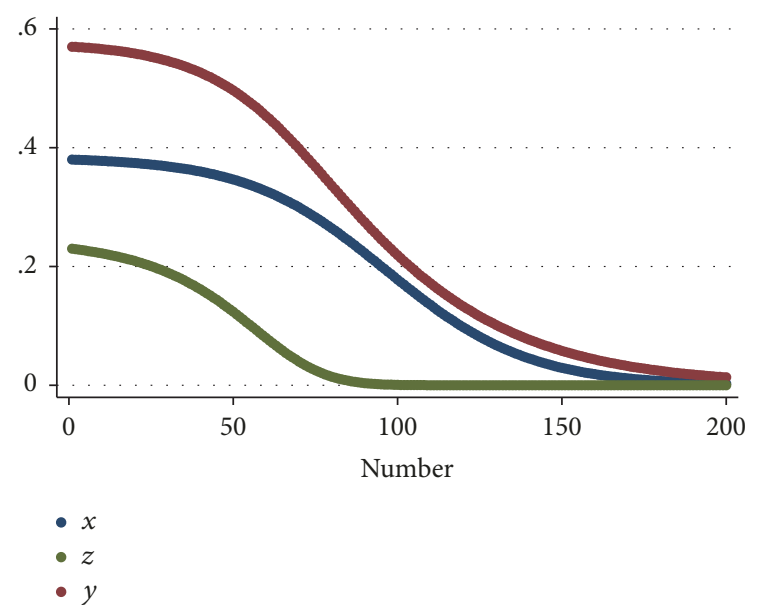

(b)

FIGURE 3: Simulation results when initial coordinates change.

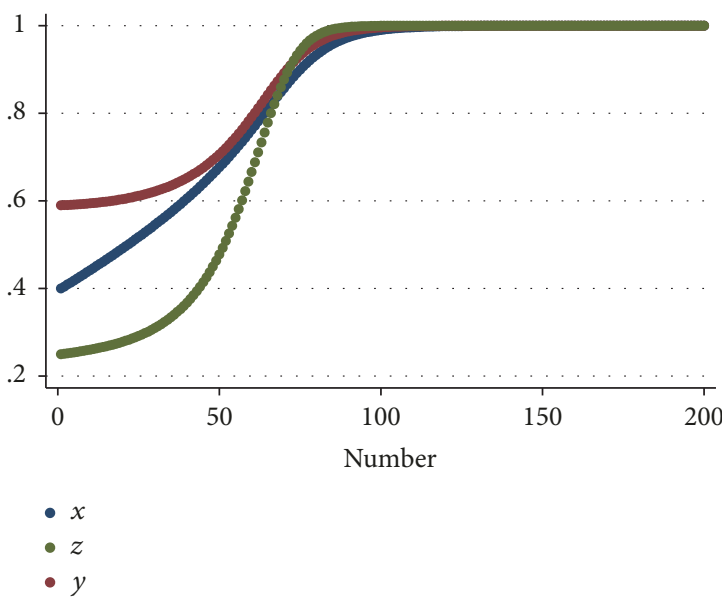

(a)

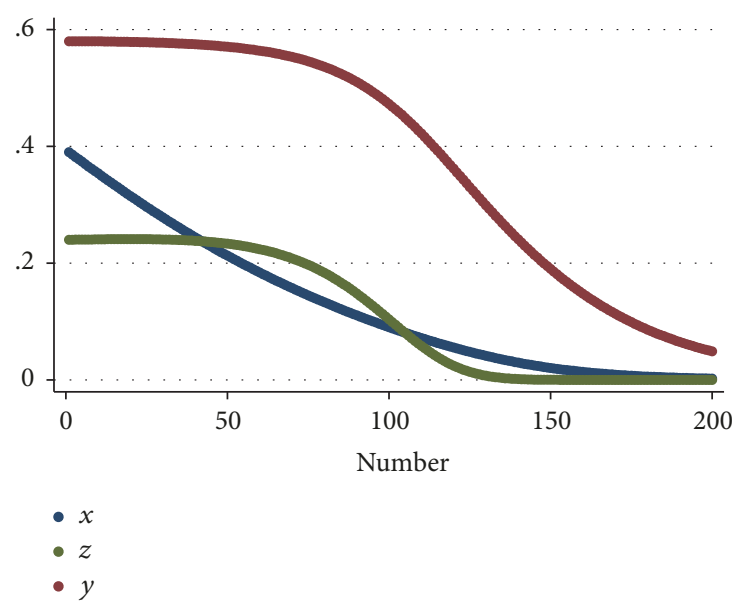

(b)

FIGURE 4: Simulation results when $d$ changes.

value of $(x, y, z)$ is set as $(0.38,0.57,0.23)$, which is slightly lower than the saddle point, the system would converge to the undesirable evolutionary stable state $(0,0,0)$, as shown in Figure $3(\mathrm{~b})$. To a great extent, this result demonstrates that the direction and speed of the evolution are decided by the initial proportions of the agents' strategic choices. When the initial proportions are quite low, the system could not converge to the desirable evolutionary stable state with a quite high possibility. For better observation and discussion, we set the initial position as $(0.40,0.59,0.25)$ and we focus on the curve's change caused by the variation of some key parameters.

Figure 4 shows the simulation results when the parameter $d$ changes but the values of other parameters remain unchanged. When $d$ rises, the marketers increase the punishment strength on the farmers for farmers' unilateral violation of the quality criteria, and vice versa. In Figure 4(a), when $d$ increases from 0.90 to 1.20 , the system converges to the evolutionary stable state $(1,1,1)$ faster than in the initial condition. But in Figure 4(b), when $d$ decreases from 0.90 to
0.60 , the system converges to the undesirable evolutionary stable state $(0,0,0)$ instead after 200 iterations. Because the punishment on farmers for unilateral violation of the quality criteria is low, the opportunity costs for farmers to produce low-quality agri-food are low, and the farmers are more motivated to take opportunistic behaviors.

Figure 5 shows the simulation results when the parameter $r$ changes but the values of other parameters remain unchanged. When $r$ rises, the market obtains more premium via selling high-quality agri-food due to quality certification, customer satisfaction, customer loyalty, and so forth, and vice versa. In Figure 5(a), when $r$ increases from 0.19 to 0.45 , the system converges to the evolutionary stable state $(1,1$, 1) faster than in the initial condition. But in Figure 5(b), when $r$ decreases from 0.19 to 0.05 , the system converges to the evolutionary stable state $(0,0,0)$. Ceteris paribus, when the market quality premium increases, the evolutionary system converges to desirable stability faster, but when the market quality premium decreases, the evolutionary system 


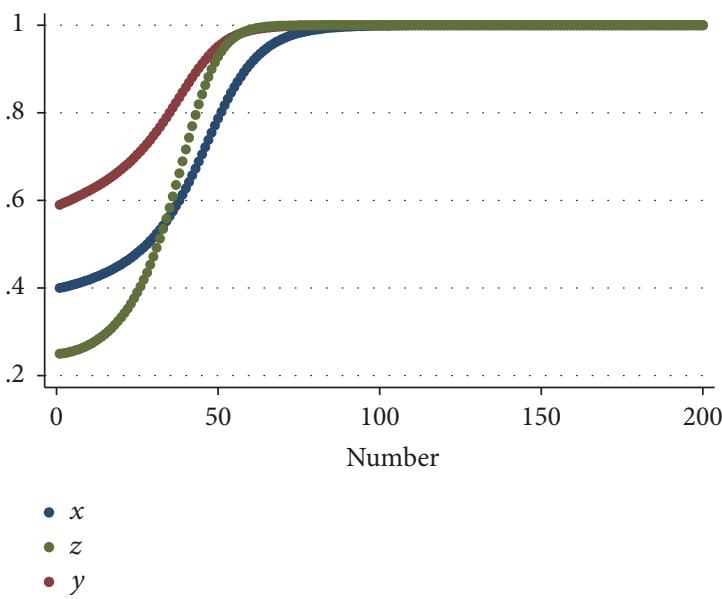

(a)

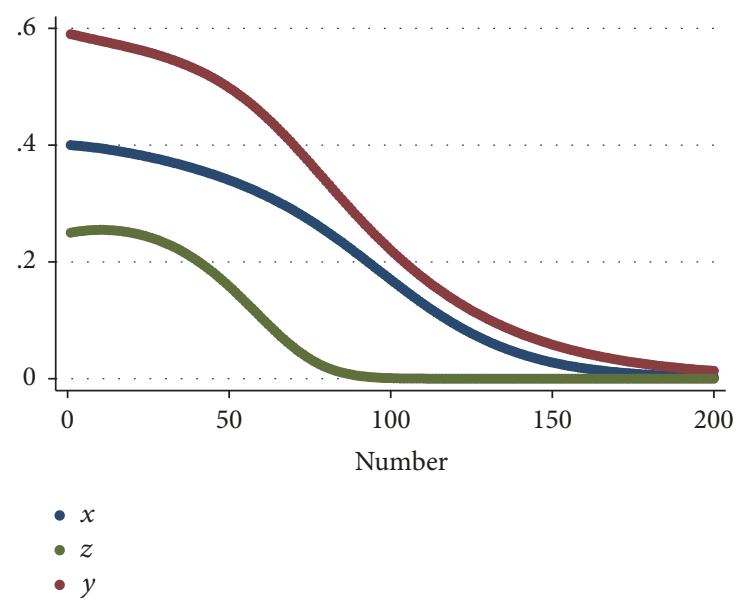

(b)

FIgURE 5: Simulation results when $r$ changes.

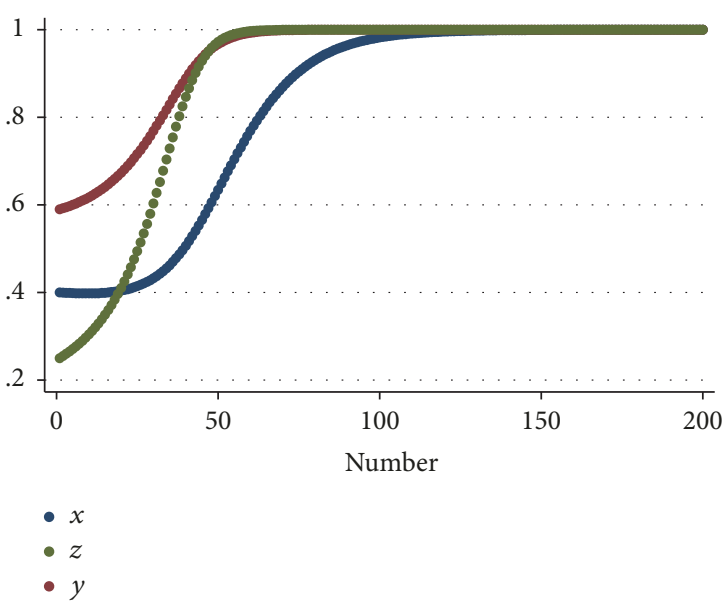

(a)

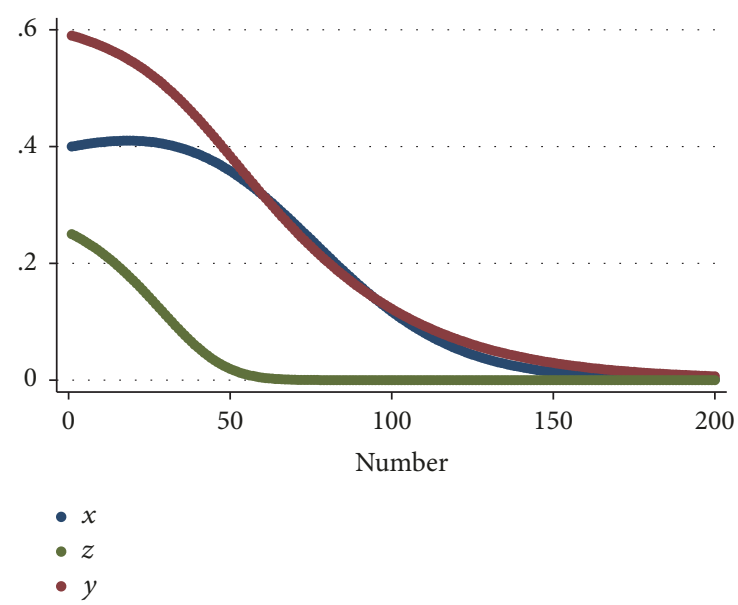

(b)

FIGURE 6: Simulation results when $k$ changes.

converges to desirable stability slower or even converges to the undesirable stability. To some extent, the market premium is decided by consumers' trust for agri-food quality, which could be improved enormously by the information traceability mechanism.

Figure 6 shows the simulation results when the parameter $k$ changes but the values of other parameters remain unchanged. When $k$ rises, the mathematical expectation of compensation demanded by the consumers who charge the illegal marketers increases, and vice versa. In Figure 6(a), when $k$ increases from 0.40 to 0.60 , the system converges to the evolutionary stable state $(1,1,1)$ faster than in the initial condition. But in Figure 6(b), when $k$ decreases from 0.40 to 0.20 , the system converges to the evolutionary stable state $(0$, $0,0)$. Ceteris paribus, when the mathematical expectation of compensation demanded by consumers rises, the evolutionary system converges to desirable stability faster, but when the mathematical expectation declines, the evolutionary system converges to desirable stability slower or even converges to the undesirable stability. In emerging economies, the costs of consumers for safeguarding their rights through legal approach are often extremely high, so to reduce the consumers' litigation expense might be the key factor to take full advantage of consumers' adverse supervision.

Figure 7 shows the simulation results when the parameters $\delta$ and $\varepsilon$ change but the values of other parameters remain unchanged. The increase in $\delta$ means more high-quality agri-food market premium is delivered from marketers to farmers, and vice versa. The decrease in $\varepsilon$ means that less compensation liabilities for violating the quality standards are assumed by the farmers, and vice versa. It is obvious that the proportion $x$ approaches 1 at a lower speed when $\delta$ decreases from 0.6 to 0.1 in Figure 7(a). And the system converges to the evolutionary stable state $(0,0$, and 0$)$ when $\varepsilon$ increases from 0.6 to 0.9 in Figure 7(b). Since following the high-quality standards causes high production cost, farmers rely on the distribution of high-quality premiums to make up for the 


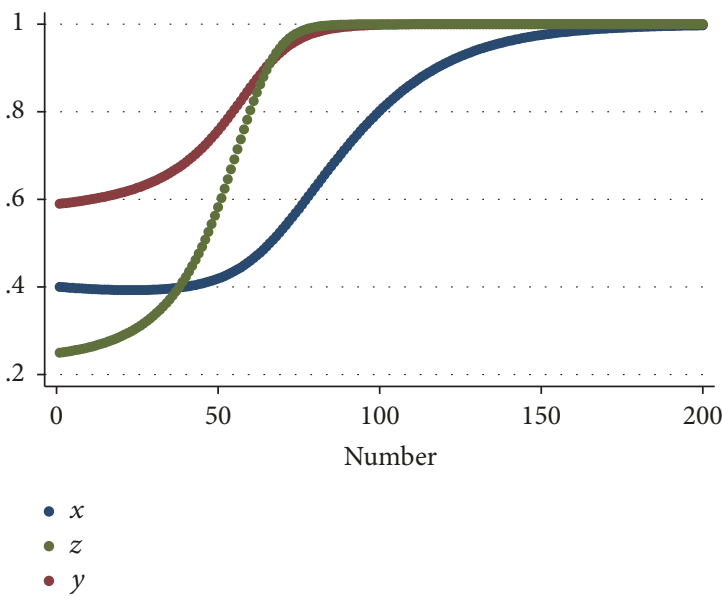

(a)

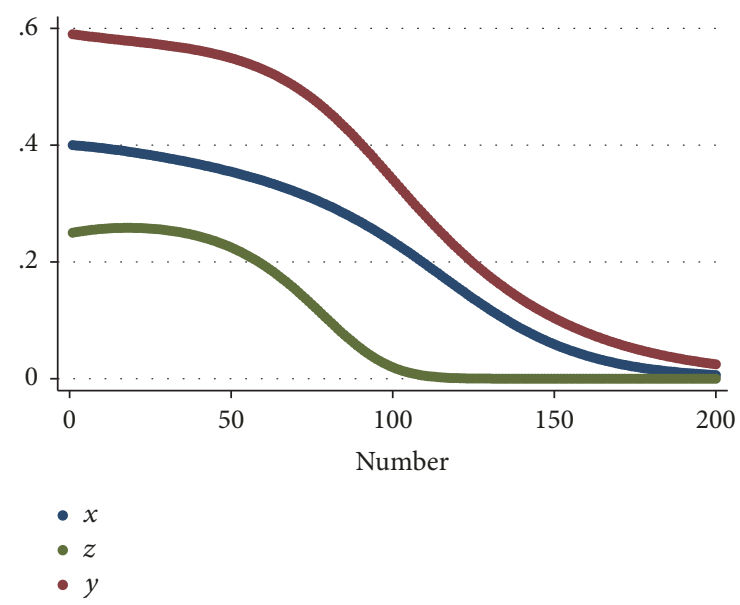

(b)

FIGURE 7: Simulation results when $\delta$ and $\varepsilon$ change.

losses. When they get more from the profit disposition, they are more motivated to produce high-quality agri-food.

\section{Conclusion}

The trilateral evolutionary game of agri-food quality in farmer-supermarket direct purchase could be seen as a nonlinear interactive process, in which each participant's decision-making influences the others' payoffs and the direction of the systematical evolution. By learning, replicating, and inheriting optimal strategies, strategies with high payoff will spread within the population, leading the system to reach a desirable or undesirable equilibrium. The evolutionary speed changes with time and depends on the individuals' adaptability, as well as the population sizes, the cooperative tendencies, and the information communication efficiency. Rules of reward and punishment determine the spontaneous order of agri-food quality promotion mechanism, making the evolutionary system converge to desirable or undesirable evolutionary stability. Moreover, when the proportions of participants who choose optimal strategies get below the saddle point or some parameters exceed some threshold, the evolutionary system has entirely different final equilibrium point.

The proportion that each group chooses strategy 1 in the initial condition determines the final evolutionary stable state. If most of farmers and marketers insufficiently recognize the significance of agri-food quality and safety, the initial proportions of the participants who take active high-quality strategies will be very low. This would make the evolutionary system converge to the undesirable evolutionary stable state. Ceteris paribus, the simulation results show that as the indemnity increases when farmers unilaterally violate the high-quality criteria, the farmers' expected revenues decrease and the evolutionary system converges to desirable stability faster, and vice versa. Moreover, high market quality premium breeds active trilateral evolutionary game of agri-food quality, and the market quality premium results in the passive evolutionary game. Furthermore, enhancing consumers' awareness, capabilities, and conditions to protect their own rights would help the trilateral evolutionary game of agrifood quality to converge to an active evolutionary stable state. In addition, the advancement of premium sharing and the reduction of compensation transferring would encourage farmers to take more active production behavior to maintain the agri-food quality.

Therefore, the evolutionary system converges to desirable stability faster, when the high-quality agri-food's market premium increases and the penalty for violating quality standards increases. Furthermore, when farmers share more high-quality agri-food's market premiums and marketers compensate more for violating the quality standards than before, the evolutionary system also converges to desirable stability faster. Therefore, the quality information traceability technology, farmers and marketers' fair distribution of profits and risks, and consumers' capabilities to safeguard their legal rights are the three key factors to maintain the effectiveness and stability of quality assurance systems.

\section{Conflicts of Interest}

The authors declare no conflicts of interest.

\section{Acknowledgments}

This paper is supported by the China National Philosophy and Social Science as an important program (15AGL014) and the Development Program for Science and Technology Research of Shandong Province (2016GSF20013).

\section{References}

[1] X. Su, Y. Wang, S. Duan, and J. Ma, "Detecting chaos from agricultural product price time series," Entropy, vol. 16, no. 12, pp. 6415-6433, 2014. 
[2] J. Ma and B. Bao, "Research on bullwhip effect in energyefficient air conditioning supply chain," Journal of Cleaner Production, vol. 143, pp. 854-865, 2017.

[3] J. Ma, T. Li, and W. Ren, "Research on the Complexity of DualChannel Supply Chain Model in Competitive Retailing Service Market," vol. 27, 2017.

[4] A. Zago, "A nonparametric analysis of production models with multidimensional quality," American Journal of Agricultural Economics, vol. 91, no. 3, pp. 751-764, 2009.

[5] A. Maffioli, D. Ubfal, G. V. Baré, and P. Cerdán-Infantes, "Extension services, product quality and yields: The case of grapes in Argentina," Agricultural Economics, vol. 42, no. 6, pp. 727-734, 2011.

[6] C. Saenger, M. Qaim, M. Torero, and A. Viceisza, "Contract farming and smallholder incentives to produce high quality: Experimental evidence from the Vietnamese dairy sector," Agricultural Economics (United Kingdom), vol. 44, no. 3, pp. 297-308, 2013.

[7] M.-L. Rau and F. Van Tongeren, "Modeling differentiated quality standards in the agri-food sector: The case of meat trade in the enlarged EU," Agricultural Economics, vol. 37, no. 2-3, pp. 305-315, 2007.

[8] J. A. Winfree and J. J. McCluskey, "Collective reputation and quality," American Journal of Agricultural Economics, vol. 87, no. 1, pp. 206-213, 2005.

[9] A. E. Saak, "Collective reputation, social norms, and participation," American Journal of Agricultural Economics, vol. 94, no. 3 , pp. 763-785, 2012.

[10] E. Galdeano, "An inverse demand analysis with introduction of quality effects: An application to Spanish consumption of fruit and vegetables," Agricultural Economics, vol. 33, no. 2, pp. 163$177,2005$.

[11] Z. Gao, X. Yu, and J.-Y. Lee, "Consumer demand for diet quality: Evidence from the healthy eating index," Australian Journal of Agricultural and Resource Economics, vol. 57, no. 3, pp. 301-319, 2013.

[12] G. T. Tonsor, "Consumer inferences of food safety and quality," European Review of Agricultural Economics, vol. 38, no. 2, pp. 213-235, 2011.

[13] M. Fafchamps, R. V. Hill, and B. Minten, "Quality control in nonstaple food markets: Evidence from India," Agricultural Economics, vol. 38, no. 3, pp. 251-266, 2008.

[14] J. J. Mccluskey, "A Game Theoretic Approach To Organic Foods: An Analysis Of Asymmetric Information And Policy," Agricultural Resource Economics Review, vol. 29, no. 1, p. 1, 2000.

[15] R. Hoffmann, "Ownership Structure and Endogenous Quality Choice: Cooperatives versus Investor-Owned Firms," Journal of Agricultural Food Industrial Organization, vol. 3, no. 2, p. 26, 2005.

[16] P. Fousekis, "Quality choices in a vertical structure: national brands vs private labels in grocery retailing," Agricultural Economics Review, vol. 11, no. 2, pp. 34-43, 2010.

[17] S. Guo, J. Ma, and X. Zhan, "Complexity and Application of Tobacco Manufacturer Pricing Game considering Market Segments," Discrete Dynamics in Nature and Society, vol. 2017, pp. 1-11, 2017.

[18] J. Ma and X. Ma, "Measure of the bullwhip effect considering the market competition between two retailers," International Journal of Production Research, vol. 55, no. 2, pp. 1-14, 2016.

[19] J. Ma and H. Wang, "Complexity analysis of dynamic noncooperative game models for closed-loop supply chain with product recovery," Applied Mathematical Modelling, vol. 38, no. 23, pp. 5562-5572, 2014.

[20] J. Ma, W. Yang, and W. Lou, "Research on Bifurcation and Chaos in a Dynamic Mixed Game System with Oligopolies under Carbon Emission Constraint," International Journal of Bifurcation and Chaos, vol. 27, no. 10, Article ID 1750158, 2017.

[21] J. W. Weibull, Evolutionary Game Theory, The MIT Press, Cambridge, Mass, USA, 1995.

[22] J. Ma and Z. Guo, "The parameter basin and complex of dynamic game with estimation and two-stage consideration," Applied Mathematics and Computation, vol. 248, pp. 131-142, 2014.

[23] Y. Wang, X. Su, and S. Guo, "The optimal confidence intervals for agricultural products' price forecasts based on hierarchical historical errors," Entropy, vol. 18, no. 12, article no. 439, 2016. 


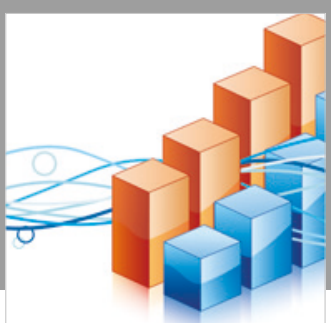

Advances in

Operations Research

\section{-n-m}
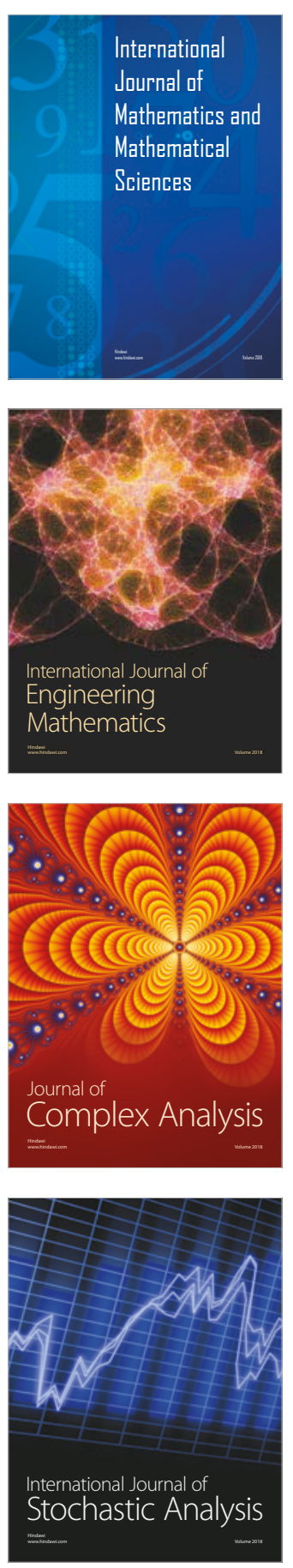
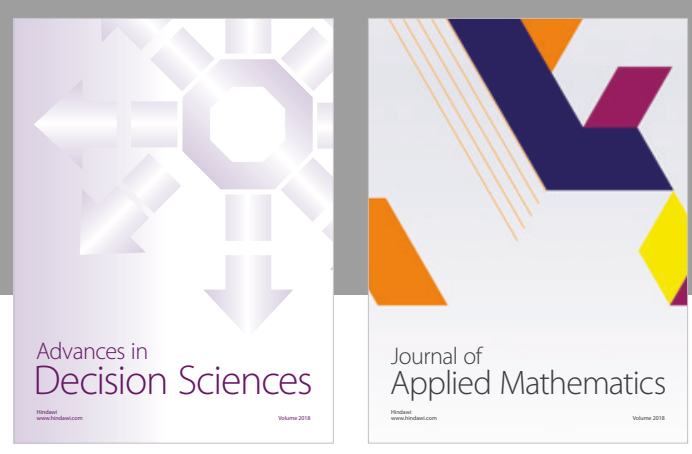

Journal of

Applied Mathematics
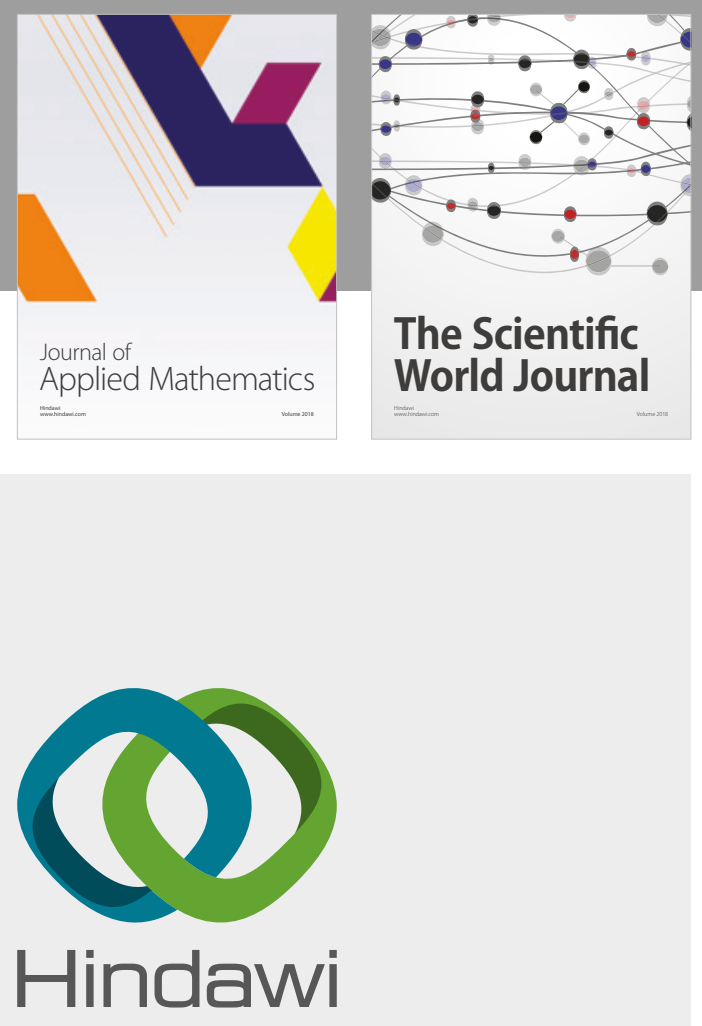

Submit your manuscripts at

www.hindawi.com

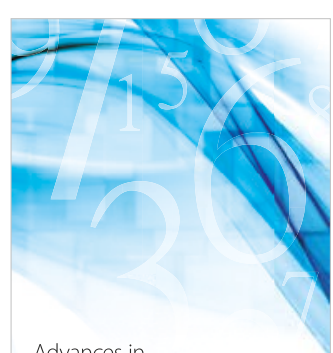

Advances in
Numerical Analysis
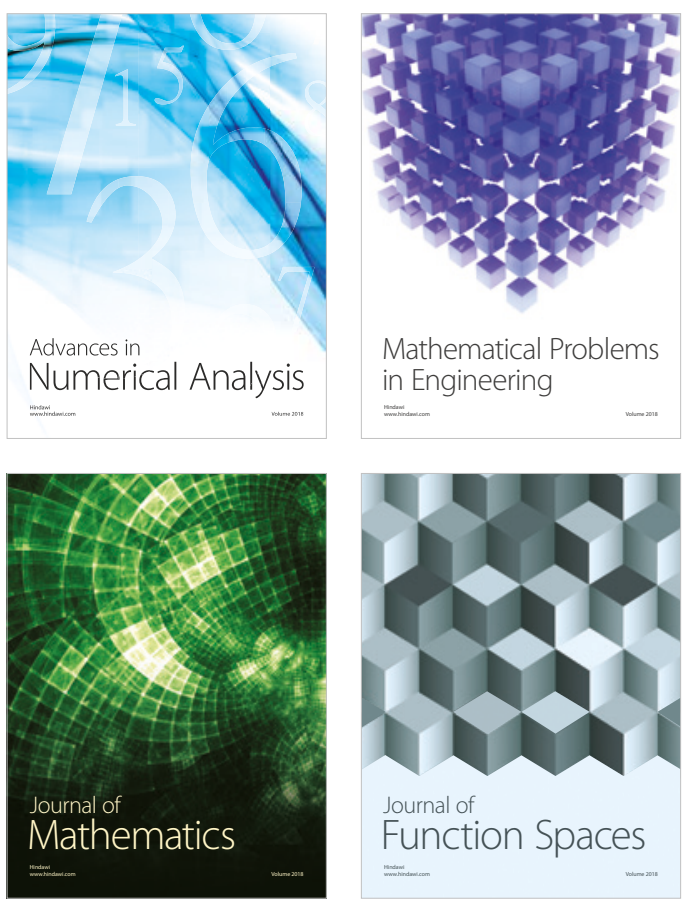

Mathematical Problems in Engineering

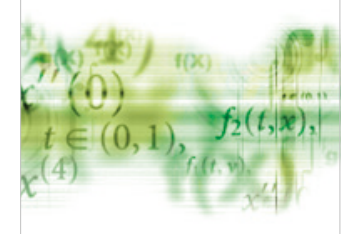

International Journal of

Differential Equations

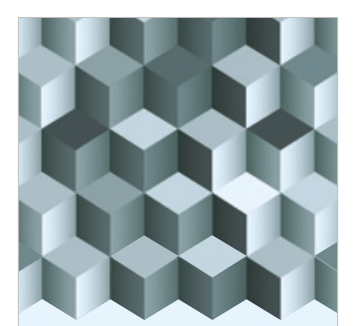

Journal of

Function Spaces
The Scientific

World Journal

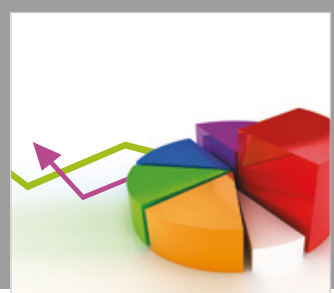

Journal of

Probability and Statistics
